\title{
A characterization of the finite Veronesean by intersection properties
}

\author{
J. Schillewaert, J.A. Thas and H. Van Maldeghem \\ Department of Pure Mathematics and Computer Algebra, \\ Ghent University \\ Krijgslaan 281-S22, B-9000 Gent, Belgium \\ \{jschille, jat, hvm\}@cage.ugent.be
}

October 8, 2008

\begin{abstract}
A combinatorial characterization of the Veronese variety of all quadrics in $\mathbf{P G}(n, q)$ by means of its intersection properties with respect to subspaces is obtained. The result relies on a similar combinatorial result on the Veronesean of all conics in the plane PG $(2, q)$ by Ferri [2], Hirschfeld and Thas [4], and Thas and Van Maldeghem [7], and a structural characterization of the quadric Veronesean by Thas and Van Maldeghem [6].
\end{abstract}

\section{Introduction}

An important branch in combinatorics is the characterization of algebraically defined objects in a combinatorial way. In several situations, it might occur that one has information about the intersection numbers with subspaces of a certain point set $\mathcal{K}$, but no structural information. In such cases, characterization results classifying the possible structures having these properties can be very useful. In this paper, we characterize the finite Veronese variety by means of such intersection properties. For the smallest Veronesean, the conic, this was already done (in the odd case) by Segre, in his celebrated characterization of conics ("every set of $q+1$ points in $\mathbf{P G}(2, q), q$ odd, no three of which are collinear, is a conic") [5]. This was in fact the starting point of this kind of results. For the Veronese surface of all conics in PG(2,q), it was already done Ferri [2], Hirschfeld and Thas [4], and Thas and Van Maldeghem [7].

Definition The Veronese variety $\mathcal{V}_{n}^{2^{n}}$ of all quadrics of $\mathbf{P G}(n, q), n \geq 1$ is the variety $\mathcal{V}_{n}^{2^{n}}=\left\{p\left(x_{0}^{2}, x_{1}^{2}, \cdots, x_{n}^{2}, x_{0} x_{1}, x_{0} x_{2}, \cdots, x_{n-1} x_{n}\right) \mid\left(x_{0}, \cdots, x_{n}\right)\right.$ is a point of $\left.\mathbf{P G}(n, q)\right\}$

of $\mathbf{P G}\left(\frac{n(n+3)}{2}, q\right)$; this variety has dimension $n$ and order $2^{n}$. The natural number $n$ is called the index of $\mathcal{V}_{n}^{2^{n}}$. 
For the basic properties of Veroneseans we refer to [4]. Here we only mention the following, stating that one can also consider the Veronesean from a matrix point of view.

Theorem 1.1 The quadric Veronesean $\mathcal{V}_{n}^{2^{n}}$ of $\mathbf{P G}(n, q)$ consists of all points $p\left(y_{0,0}, \cdots, y_{n-1, n}\right)$ of $\mathbf{P G}(N, q)$ for which rank $\left[y_{i j}\right]=1$.

In [6] the following characterization of the finite quadric Veroneseans $\mathcal{V}_{n}^{2^{n}}$ was proved.

Theorem 1.2 Let $\mathcal{F}$ be a set of $\frac{q^{n+1}-1}{q-1} n$-dimensional spaces in $\mathbf{P G}\left(\frac{n(n+3)}{2}, q\right), n \geq 2$, with the following properties:

(VS1) Each two elements of $\mathcal{F}$ intersect in a point.

(VS2) Each three elements of $\mathcal{F}$ are skew.

(VS3) The elements of $\mathcal{F}$ span $\mathbf{P G}\left(\frac{n(n+3)}{2}, q\right)$.

(VS4) Any proper subspace of $\mathbf{P G}\left(\frac{n(n+3)}{2}, q\right)$ that is spanned by a collection of elements of $\mathcal{F}$ is a subspace of dimension $\frac{i(2 n-i+3)}{2}-1$ for some $i \in\{0, \ldots, n\}$.

(VS5) If $q$ is even, at least one space spanned by two elements of $\mathcal{F}$ contains more than two elements.

Then either $\mathcal{F}$ is the set of tangent spaces of a quadric Veronesean $\mathcal{V}_{n}^{2^{n}}$ or $q$ is even, there are two members $\Omega_{1}, \Omega_{2} \in \mathcal{F}$ such that the $2 n$-dimensional space $\left\langle\Omega_{1}, \Omega_{2}\right\rangle$ only contains 2 elements of $\mathcal{F}$ and there is a unique subspace $\Omega$ of dimension $n$ such that $\Omega \cup \mathcal{F}$ is the set of tangent subspaces together with the nucleus subspace of a quadric Veronesean $\mathcal{V}_{n}^{2^{n}}$. In particular, if $n=2$, then the statement holds under the weaker hypothesis of $\mathcal{F}$ satisfying (VS1), (VS2), (VS3) and (VS5).

For $n=1$ and $q$ even every dual oval satisfies (VS1) - (VS5). For $n=2$ one can classify all examples that do not satisfy (VS5) by a result of [1], and the only possibilities are $q=2$ and $q=4$. This classification remains open for $n \geq 3$, although an infinite class of examples is known for $q=2$, see [6].

In particular for $n=2$, this result generalizes Theorem 25.2.14 of [4] to $q$ even, and allows to generalize Theorem 25.3.14 of [4] to $q$ even, and so we obtain

Theorem 1.3 If $\mathcal{K}$ is a set of $k$ points of $\mathbf{P G}(5, q), q \neq 2,4$, which satisfies the following conditions

(i) $\left|\Pi_{4} \cap \mathcal{K}\right|=1, q+1,2 q+1$ for every hyperplane $\Pi_{4}$ of $\mathbf{P G}(5, q)$ and there exists a hyperplane $\Pi_{4}$ for which $\left|\Pi_{4} \cap \mathcal{K}\right|=2 q+1$.

(ii) Any plane of $\mathbf{P G}(5, q)$ with four points in $\mathcal{K}$ has at least $q+1$ points in $\mathcal{K}$. 
Then $\mathcal{K}$ is the point set of a Veronesean $\mathcal{V}_{2}^{4}$.

A theorem by Zanella [8] gives an upper bound for the intersection of $k$-dimensional subspaces with the quadric Veronese variety, so for the intersections $\Pi_{k} \cap \mathcal{V}_{n}$.

Theorem 1.4 Consider the Veronesean surface defined by the mapping

$$
\begin{gathered}
\zeta: \mathbf{P G}(n, q) \rightarrow \mathbf{P G}\left(\frac{n(n+3)}{2}, q\right), \\
\left(x_{0}, x_{1}, \cdots, x_{n}\right) \rightarrow\left(x_{0}^{2}, x_{1}^{2}, \cdots, x_{n-1} x_{n}\right) .
\end{gathered}
$$

If $k$, a are natural numbers such that $k+1 \leq \frac{(a+3)(a+2)}{2}$, then the intersections $\Pi_{k} \cap \mathcal{V}_{n}$ contain at most

$$
\frac{q^{a+1}-1}{q-1}+q^{k-\frac{(a+2)(a+1)}{2}}
$$

points.

Applying this for small dimensions yields the upper bounds $q+1, q+2,2 q+1$ and $q^{2}+q+1$ for $k=2, k=3, k=4$ and $k=5$ respectively.

A result of the second and third author [7] of this paper characterizes Veronese varieties in terms of ovals.

Theorem 1.5 Let $X$ be a set of points in $\Pi:=\mathbf{P G}(M, q), M>2$, spanning $\Pi$, and let $\mathcal{P}$ be a collection of planes such that for any $\pi \in \mathcal{P}$, the intersection $X \cap \pi$ is an oval in $\pi$. For $\pi \in \mathcal{P}$ and $x \in X \cap \pi$, we denote by $T_{x}(\pi)$ the tangent line to $X \cap \pi$ at $x$ in $\pi$. We assume the following three properties.

(i) Any two points $x, y \in X$ lie in a unique member of $\mathcal{P}$ which we denote by $[x, y]$.

(ii) If $\pi_{1}, \pi_{2} \in \mathcal{P}$ and $\pi_{1} \cap \pi_{2}$ is non-empty then $\pi_{1} \cap \pi_{2} \subset X$.

(iii) If $x \in X$ and $\pi \in \mathcal{P}$ with $x \notin \pi$, then each of the lines $T_{x}([x, y]), y \in X \cap \pi$, is contained in a plane of $\Pi$, denoted by $T(x, \pi)$.

Then there exists a natural number $n \geq 2$ (called the index of $X$ ), a projective space $\Pi^{\prime}:=\mathbf{P G}\left(\frac{n(n+3)}{2}, q\right)$ containing $\Pi$, a subspace $R$ of $\Pi^{\prime}$ skew to $\Pi$, and a quadric Veronesean $\mathcal{V}_{n}$ of index $n$ in $\Pi^{\prime}$, with $R \cap \mathcal{V}_{n}=\emptyset$, such that $X$ is the (bijective) projection of $\mathcal{V}_{n}$ from $R$ onto $\Pi$. The subspace $R$ can be empty, in which case $X$ is projectively equivalent to $\mathcal{V}_{n}$.

To conclude this introduction, we define $k$-arcs in $\mathbf{P G}(3, q)$.

Definition A $k$-arc of $\mathbf{P G}(3, q), k \geq 4$ is a set of $k$ points, no 4 of which are coplanar.

If $q \geq 5$, by Theorem 21.2.4 and Theorem 21.3.8 of [3] we know that $k \leq q+1$. 


\section{First characterization}

We want to use the following set of conditions to characterize the quadric Veronesean. Consider a set $\mathcal{K}$ of $\frac{q^{n+1}-1}{q-1}$ points spanning $\mathbf{P G}\left(\frac{n(n+3)}{2}, q\right)$, with $n \geq 2$, such that

(P) If a plane intersects $\mathcal{K}$ in more than three points then it contains exactly $q+1$ points of $\mathcal{K}$. Furthermore, any two points $p_{1}, p_{2}$ of $\mathcal{K}$ are contained in a plane containing $q+1$ points of $\mathcal{K}$.

(S) If a 3 -space $\Pi_{3}$ intersects $\mathcal{K}$ in more than 4 points then there are four points of $\mathcal{K}$ contained in a plane of $\Pi_{3}$. In particular, by (i), this implies that if $\left|\Pi_{3} \cap \mathcal{K}\right|>4$, then $\left|\Pi_{3} \cap \mathcal{K}\right| \geq q+1$.

(V) If a 5 -space $\Pi_{5}$ intersects $\mathcal{K}$ in more than $2 q+2$ points then it intersects $\mathcal{K}$ in exactly $q^{2}+q+1$ points.

Remark. Planes intersecting $\mathcal{K}$ in $q+1$ points and 5 -spaces intersecting $\mathcal{K}$ in $q^{2}+q+1$ points will be called big planes and big 5-spaces respectively. Assume $q \geq 5$ in the following.

We will prove the following main theorem.

Theorem 2.1 If $q \geq 5$, then the set $\mathcal{K}$ is the point set of the Veronese variety of all quadrics of $\mathbf{P G}(n, q)$.

\section{Remark.}

A counterexample for $q=2$ to the previous theorem is given by destructing a Veronese variety by removing one point of it and replacing it by a point in projective space which corresponds with a matrix of maximal rank, see Theorem 1.1.

A counterexample for $q=3, n=2$ is given by the point set formed by the points of an elliptic quadric $\mathcal{E}$ lying in a space $\Pi_{3}$ and 3 points on a line $l$ which does not intersect $\Pi_{3}$.

First of all we have to prove that these conditions are well-chosen, meaning the object we want to characterize satisfies them.

Theorem 2.2 The Conditions (P), $(S)$ and $(V)$ above hold for the Veronesean $\mathcal{V}_{n}^{2^{n}}$.

Proof For Condition (P), we cannot use Lemma 25.3.1 of [4] directly, since we don't know a priori that every plane is contained in a 5 -space intersecting $\mathcal{K}$ in a $\mathcal{V}_{2}^{4}$ but a slight adaptation of the argument works. Suppose that the plane $\pi$ contains at least four distinct points $q_{1}, q_{2}, q_{3}, q_{4}$ of $\mathcal{V}_{n}^{2^{n}}$. By Corollary 1 of Theorem 25.1 .9 of [4], the points $q_{i}, q_{j}$, with $i \neq j$, are contained in a unique conic of $\mathcal{V}_{n}^{2^{n}}$. Let $C^{\prime}$, in the plane $\pi^{\prime}$, be the conic defined by $q_{1}$ and $q_{2}$, and, let $C^{\prime \prime}$, in the plane $\pi^{\prime \prime}$, be the conic defined by $q_{2}$ and $q_{3}$. Suppose that $C^{\prime} \neq C^{\prime \prime}$. By Theorem 1.4 the conic planes $\pi^{\prime}$ and $\pi^{\prime \prime}$ generate a 4 -space $\Pi_{4}$ such that $\left|\Pi_{4} \cap \mathcal{K}\right| \leq 2 q+1$. But besides the $2 q+1$ points in 
$C^{\prime} \cup C^{\prime \prime}$, the point $q_{4}$ would also be contained in this 4-space, a contradiction. Hence $|\Pi \cap \mathcal{K}| \geq q+1$ and by Theorem 1.4, $|\Pi \cap \mathcal{K}|=q+1$. Conditions (S) and (V) can be proved using a coordinatization and checking the different possibilities for the position of the inverse images of the points in $\mathbf{P G}(n, q)$.

We prove some upper bounds for the number of points of $\mathcal{K}$ contained in low-dimensional spaces.

Lemma 2.3 If $n>2$, every 4 -space contains at most $2 q+2$ points of $\mathcal{K}$.

Proof Let $\Pi$ be a 4-space. By Condition (V), it follows directly that $|\Pi \cap \mathcal{K}| \leq q^{2}+q+1$ and clearly $|\Pi \cap \mathcal{K}|=q^{2}+q+1$ also yields a contradiction.

Suppose that $2 q+2<|\Pi \cap \mathcal{K}|<q^{2}+q+1$. Again by Condition (V), every 5 -space through $\Pi$ contains exactly $q^{2}+q+1$ points. The number of 5 -spaces through a fixed 4-space in $\mathbf{P G}\left(\frac{n(n+3)}{2}, q\right)$ is equal to $\frac{q^{\frac{n(n+3)}{2}-4}-1}{q-1}$. Hence, we get at least

$$
\frac{q^{\frac{n(n+3)}{2}-4}-1}{q-1}+2 q+2>\frac{q^{n+1}-1}{q-1}
$$

points in $|\mathcal{K}|$, a contradiction since $n>2$.

Lemma 2.4 Any line $l$ meets $\mathcal{K}$ in at most 2 points. Hence, a plane $\pi$ with $|\pi \cap \mathcal{K}|=$ $q+1$ intersects $\mathcal{K}$ in an oval.

Proof First suppose that $|l \cap \mathcal{K}|=3$. If $n>2$, then consider 3 planes $\pi_{1}, \pi_{2}, \pi_{3}$ through $l$ containing more than 3 points of $\mathcal{K}$ and hence by Condition $(\mathrm{P}) q+1$ points of $\mathcal{K}$. Then $\operatorname{dim}\left\langle\pi_{1}, \pi_{2}, \pi_{3}\right\rangle \leq 4$. For $q>5$, this yields a contradiction by Lemma 2.3. If $q=5$, then consider a 3 -space $\Pi_{3}$ through $l$ containing at least 9 points of $\mathcal{K}$ inside a big 5 -space $\Pi_{5}$. But then considering all 4-spaces through $\Pi_{3}$ inside $\Pi_{5}$, by Lemma 2.3 , we get at most $6 \cdot 3+9=27$ points in $\Pi_{5} \cap \mathcal{K}$, a contradiction.

If $n=2$ then we get the following equation for the number $\alpha$ of planes through $l$ which contain exactly $q+1$ points of $\mathcal{K}$ :

$$
\alpha(q-2)+3=q^{2}+q+1 .
$$

This yields a contradiction if $q \geq 5$. Next, suppose that $|l \cap \mathcal{K}|=x$, with $3<x<q+1$. Consider all planes through $l$. Then clearly, we get too many points for our set $\mathcal{K}$, a contradiction. Finally, if $|l \cap \mathcal{K}|=q+1$, we also get a contradiction as planes can contain at most $q+1$ points of $\mathcal{K}$.

The previous lemma allows us for $n=2$ to prove the same upper bound as in Lemma 2.3 .

Lemma 2.5 Every 4-space intersects $\mathcal{K}$ in at most $2 q+2$ points. Hence, every 3 -space contained in a big 5 -space intersects $\mathcal{K}$ in at most $q+3$ points. 
Proof For $n>2$ this is Lemma 2.3. Next let $n=2$.

Suppose there exists a 3 -space $\Pi_{3}$ which contains two planes $\pi_{1}$ and $\pi_{2}$ which intersect $\mathcal{K}$ in ovals $\mathcal{O}_{1}$ and $\mathcal{O}_{2}$ respectively which have two points $p_{1}, p_{2}$ of $\mathcal{K}$ in common. Consider two points $r_{1}$ and $r_{2}$, different from $p_{1}$ and $p_{2}$, which lie on $\mathcal{O}_{1}$ and $\mathcal{O}_{2}$ respectively. Then there are most 4 planes through the line $\left\langle r_{1}, r_{2}\right\rangle$ which are not $(q+1)$-planes, namely the planes containing either the point $p_{1}$ or $p_{2}$ or those which intersect $\pi_{i}$ in a tangent line to $\mathcal{O}_{i}$ at $r_{i}$ for $i=1$ or $i=2$.

Hence, we get at least

$$
2+(q-3)(q-1)+4=q^{2}-4 q+9
$$

points in $\Pi_{3} \cap \mathcal{K}$.

The bound above is already sufficient for the rest of the proof if $q>5$. But since we now know there is a point in $\Pi_{3} \cap \mathcal{K}$ not contained in $\mathcal{O}_{1} \cup \mathcal{O}_{2}$ we can consider all planes through the line $\left\langle p, p_{1}\right\rangle$ inside $\Pi_{3}$. In this case we only get three exceptions hence we get at least

$$
2+3+(q-2)(q-1)=q^{2}-3 q+7
$$

points in $\Pi_{3} \cap \mathcal{K}$.

If one would carry out this argument a bit more carefully one can get up to $q^{2}+1$ points in $\Pi_{3} \cap \mathcal{K}$, and hence this intersection is an ovoid. However this thus not shorten the reasonings made in the rest of this proof.

Hence if there are three such 3-spaces we distinguish the following cases.

Case (i): Any two of them only intersect in a line. Then the union of the 3 -spaces contains at least $3\left(q^{2}-3 q+7\right)-3 \cdot 2$ points of $\mathcal{K}$, a contradiction since $q \geq 5$.

Case (ii): There are two of them which intersect in a plane. Then we get a 4 -space $\Pi_{4}$ containing at least $2\left(q^{2}-3 q+7\right)-(q+1)=2 q^{2}-7 q+13$ points of $\mathcal{K}$. Consider a point $p$ in $\mathcal{K}$ not contained in $\Pi_{4}$. Through $p$ and any point $r$ in $\Pi_{4} \cap \mathcal{K}$ there passes an oval of $\mathcal{K}$ by Condition (P). If none of these ovals have two points of $\mathcal{K}$ in common, we get too many points, a contradiction. If two of these ovals have two points of $\mathcal{K}$ in common then the 3 -space spanned by these two ovals contains at least $q^{2}-3 q+7$ points of $\mathcal{K}$. Hence, we get at least

$$
2 q^{2}-7 q+13+q^{2}-3 q+7-(q+1)
$$

points in $\mathcal{K}$, a contradiction since $q \geq 5$.

If there are exactly one or two such 3 -spaces we consider a 4 -space $\Pi_{4}$ containing such a 3 -space and a point $p$ in $\mathcal{K}$ not contained in $\Pi_{4}$. Through $p$ and each point $r$ in $\Pi_{4} \cap \mathcal{K}$ there passes an oval by Condition (P). For each such point $r$ we choose exactly one such oval. It can occur at most $q+1$ times that these ovals have two points of $\mathcal{K}$ in common. Hence, we clearly get too many points in $\mathcal{K}$, a contradiction.

Now consider a 4 -space $\Pi_{4}$ which intersects $\mathcal{K}$ in $x$ points. Consider a point $p$ of $\mathcal{K}$ not in $\Pi_{4}$. By Condition $(\mathrm{P})$ through every 2 points there passes an oval. Consider 
all ovals through $p$ and a point $r$ of $\Pi_{4} \cap \mathcal{K}$. Any two of these ovals can intersect in at most one point, hence we get the following equation,

$$
\frac{x}{2}(q-2)+x+1 \leq q^{2}+q+1 .
$$

This yields $x \leq 2 q+2$.

Consider a 3 -space $\Pi_{3}$ in a big 5 -space $\Pi_{5}$ which intersects $\mathcal{K}$ in $q+3+y$ points. Since all 4 -spaces through $\Pi_{3}$ inside $\Pi_{5}$ intersect $\mathcal{K}$ in at most $2 q+2$ points we get the following inequality

$$
(q+1)(q-1-y)+q+3+y \geq q^{2}+q+1 .
$$

This implies $y \leq 0$.

Now we are able to lower the bound of Lemma 2.5.

Lemma 2.6 (i) Every 4 -space $\Pi_{4}$ intersecting $\mathcal{K}$ in more than $q+1$ points contains a plane which intersects $\mathcal{K}$ in an oval.

(ii) Every 4-space $\Pi$ contains at most $2 q+1$ points of $\mathcal{K}$.

Proof (i) Suppose that $\left|\Pi_{4} \cap \mathcal{K}\right|>q+1$. Since $q \geq 5$, by a result on arcs, namely Theorem 27.6.3 of [4], there are 5 points which are contained in a 3 -space. By Condition $(\mathrm{S})$, it follows that there are 4 of them which are contained in a plane $\pi$. Hence, by Lemma 2.4, $\pi$ intersects $\mathcal{K}$ in an oval.

(ii) Suppose that $\left|\Pi_{4} \cap \mathcal{K}\right|=2 q+2$. Consider a plane $\pi$ in $\Pi_{4}$ intersecting $\mathcal{K}$ in an oval $\mathcal{O}$. Such a plane always exists by (i).

Consider 2 points $a$ and $b$ in $\Pi_{4} \cap \mathcal{K}$ but not in $\pi$ such that $\langle a, b\rangle \cap \pi=\emptyset$. Note that this is always possible, otherwise all $2 q+2$ points of $\mathcal{K}$ are contained in a 3 -space, a contradiction by Lemma 2.5. In fact, since $\Pi_{4}$ is contained in a big 5 -space $\Pi_{5}$ at most $q+3$ points of $\mathcal{K} \cap \Pi_{4}$ can be contained in a 3-space by Lemma 2.5.

Consider a third point $c$ in $\Pi_{4} \cap \mathcal{K}$, and let $p$ be the intersection point of $\pi$ and $\pi^{\prime}=\langle a, b, c\rangle$.

We distinguish the following cases.

Case (i) $: p \in \mathcal{O}$.

Since $\pi^{\prime}$ contains at least 4 points of $\mathcal{K}$ it contains at least $q+1$ points of $\mathcal{K}$ by Condition $(\mathrm{P})$. The planes $\pi$ and $\pi^{\prime}$ both intersect $\mathcal{K}$ in an oval, $\mathcal{O}$ and $\mathcal{O}^{\prime}$. Denote the remaining point in $\Pi_{4} \cap \mathcal{K}$ by $p^{\prime}$. Consider a plane $\pi^{\prime \prime}$ spanned by $p^{\prime}$ and two points $a^{\prime}$ and $b^{\prime}$ belonging to $\mathcal{O} \backslash\{p\}$. The planes $\pi^{\prime}$ and $\pi^{\prime \prime}$ intersect in a point $r$. If $r$ belongs to $\mathcal{K}$ then $\pi^{\prime \prime}$ contains at least 4 and hence by Condition $(\mathrm{P}) q+1$ points of $\mathcal{K}$. If $r$ does not belong to $\mathcal{K}$ we may assume it is not the nucleus of $\mathcal{O}^{\prime}$, otherwise we can restart the reasoning with two other points of $\mathcal{O}$. Then the 3 -space spanned by $\pi^{\prime \prime}$ and a bisecant to $\mathcal{O}^{\prime}$ through $r$, but not through $p$, contains at least 5 and hence by 
Condition (S) at least $q+1$ points. Since $q \geq 5$, in both cases we get more than $2 q+2$ points in $\Pi_{4} \cap \mathcal{K}$, a contradiction by Lemma 2.5 .

Case (ii): $p \notin \mathcal{O}$.

First of all, we assume that not all points in $\Pi_{4} \cap \mathcal{K}$ are contained in $\pi \cup \pi^{\prime}$. Since not all points in $\Pi_{4} \cap \mathcal{K}$ are contained in $\pi \cup \pi^{\prime}$, we may assume that $p$ is not the nucleus of $\mathcal{O}$. Indeed, if $p$ would be the nucleus we consider a point $c^{\prime}$ of $\Pi_{4} \cap \mathcal{K}$ not in $\pi^{\prime} \cup \pi$ and the plane $\pi^{\prime \prime}=\left\langle a, b, c^{\prime}\right\rangle$ which then intersects $\pi$ in a point $p^{\prime}$, with $p^{\prime}$ not the nucleus of $\mathcal{O}$. So in that case we continue the reasonings with $\pi^{\prime \prime}$ instead of $\pi^{\prime}$. Consider two secants of $\mathcal{O}$ through $p$, say $l$ and $l^{\prime}$. The 3 -spaces $\left\langle\pi^{\prime}, l\right\rangle$ and $\left\langle\pi^{\prime}, l^{\prime}\right\rangle$ both contain at least 5 points of $\mathcal{K}$, hence they both contain a plane intersecting $\mathcal{K}$ in an oval. These planes have to coincide, otherwise we get too many points in $\Pi_{4} \cap \mathcal{K}$. Hence, the plane $\pi^{\prime}$ intersects $\mathcal{K}$ in an oval $\mathcal{O}^{\prime}$. This yields a contradiction with the assumption at the beginning of this paragraph. Note that as a byproduct we proved that if a 4 -space contains at least $q+5$ points of $\mathcal{K}$, it contains two planes which intersect $\mathcal{K}$ in an oval, hence $\left|\Pi_{4} \cap \mathcal{K}\right| \geq 2 q+1$. Indeed we only used 4 points $a, b, c$ and $c^{\prime}$ in $\mathcal{K}$ but not in $\pi$ to find the second oval $\mathcal{O}^{\prime}$. Furthermore, these ovals can have at most one point in common, otherwise they only span a 3 -space, but a 3 -space inside $\Pi_{5}$ intersects $\mathcal{K}$ in at most $q+3$ points by Lemma 2.5 .

Next, we may suppose that $\Pi_{4} \cap \mathcal{K}$ is a union of two ovals $\mathcal{O}$ and $\mathcal{O}^{\prime}$ contained in planes $\pi$ and $\pi^{\prime}$ which intersect in a point $p$.

If $p$ is the nucleus of neither $\mathcal{O}$ or $\mathcal{O}^{\prime}$, then consider a secant $t$ of $\mathcal{O}$ and a secant $t^{\prime}$ of $\mathcal{O}^{\prime}$ through $p$. The plane spanned by $t$ and $t^{\prime}$ contains 4 and hence $q+1$ points of $\mathcal{K}$, and so $\left|\Pi_{4} \cap \mathcal{K}\right|>2 q+2$, a contradiction.

If $p$ is the nucleus of $\mathcal{O}$, but not the nucleus of $\mathcal{O}^{\prime}$, then consider a 5 -space $\Pi_{5}$ containing $\Pi_{4}$ which intersects $\mathcal{K}$ in $q^{2}+q+1$ elements. Since $p$ is not the nucleus of $\mathcal{O}^{\prime}$, there is a secant $l^{\prime}$ of $\mathcal{O}^{\prime}$ through $p$. The 3 -space $\Pi_{3}$ spanned by $\mathcal{O}$ and $l^{\prime}$ contains exactly $q+3$ elements of $\mathcal{K}$ since all 4 -spaces through $\Pi_{3}$ inside $\Pi_{5}$ contain at most $2 q+2$ elements of $\mathcal{K}$. It follows that exactly one of the 4 -spaces containing $\Pi_{3}$ in $\Pi_{5}$ intersects $\mathcal{K}$ in $2 q+1$ points, while all the other 4 -spaces through $\Pi_{3}$ in $\Pi_{5}$ contain $2 q+2$ points of $\mathcal{K}$.

By the foregoing there is a 4 -space $\Pi_{4}^{\prime} \neq \Pi_{4}$ containing $\Pi_{3}$ inside $\Pi_{5}$ which intersects $\mathcal{K}$ in $2 q+2$ elements on two ovals $\mathcal{O}$ and $\mathcal{O}^{\prime \prime}$, where the planes of $\mathcal{O}^{\prime}$ and $\mathcal{O}^{\prime \prime}$ intersect in $l^{\prime}$. Hence the 3 -space $\Pi_{3}^{\prime}$ spanned by $\mathcal{O}$ and $\mathcal{O}^{\prime \prime}$ contains at least $2 q$ elements of $\mathcal{K}$. Consider all 4 -spaces through $\Pi_{3}^{\prime}$ inside $\Pi_{5}$. By Lemma 2.5 we get at most $2 q+2(q+1)=4 q+2$ points in $\Pi_{5} \cap \mathcal{K}$, a contradiction since $q \geq 5$.

Finally, if $p$ is the nucleus of both $\mathcal{O}$ and $\mathcal{O}^{\prime}$, then consider a 3 -space $\Pi_{3}$ spanned by $\mathcal{O}$ and a tangent $l$ to $\mathcal{O}^{\prime}$ through $p$. Consider a big 5 -space $\Pi_{5}$ through $\Pi_{4}$. Consider all 4-spaces through $\Pi_{3}$ inside $\Pi_{5}$. No 4 -space through $\Pi_{3}$ inside $\Pi_{5}$ different from $\Pi_{4}$ can intersect $\mathcal{K}$ in $2 q+2$ points as well. Indeed, by Case (i) and the previous subcases of Case (ii) such a 4 -space $\Pi_{4}^{\prime}$ again has to intersect $\mathcal{K}$ in two ovals $\mathcal{O}$ and $\mathcal{O}^{\prime \prime}$, contained in planes $\pi$ and $\pi^{\prime \prime}$ respectively. The planes $\pi^{\prime}$ and $\pi^{\prime \prime}$ have to intersect in the tangent line $l$, and $p$ again has to be the nucleus of both the ovals $\mathcal{O}$ and $\mathcal{O}^{\prime \prime}$. 
But then the 3 -space $\Pi_{3}^{\prime \prime}$ spanned by $\mathcal{O}^{\prime}$ and $\mathcal{O}^{\prime \prime}$ contains at least $2 q+1$ points of $\mathcal{K}$. Consider all 4-spaces through $\Pi_{3}^{\prime \prime}$ inside $\Pi_{5}$. Then by Lemma $2.5|\Pi \cap \mathcal{K}| \leq$ $2 q+1+q+1=3 q+2$, a contradiction since $q \geq 5$.

Consider now all 4-spaces through $\Pi_{3}$ inside $\Pi_{5}$. Exactly one of them intersects $\mathcal{K}$ in $2 q+2$ points by the previous and all the others intersect $\mathcal{K}$ in at most $2 q+1$ points. Hence, by an easy inspection, there has to be a 4 -space through $\Pi_{3}$ in $\Pi_{5}$ containing exactly $2 q$ points of $\mathcal{K}$, but this yields a contradiction by the remark made at the end of the first paragraph of Case (ii).

Remark. A 4-space intersecting $\mathcal{K}$ in $2 q+1$ points will be called a big 4-space.

Lemma 2.7 (i) Inside a big 5-space $\Pi_{5}$ all 3-spaces contain at most $q+2$ points. Furthermore, all 4 -spaces inside $\Pi_{5}$ through a 3-space intersecting $\mathcal{K}$ in $q+2$ points are big ones.

(ii) $A$ big 4-space $\Pi_{4}$ contained in a big 5-space $\Pi_{5}$ intersects $\mathcal{K}$ in two ovals $\mathcal{O}_{1}, \mathcal{O}_{2}$ with $\mathcal{O}_{1} \cap \mathcal{O}_{2}=\{P\}, P \in \mathcal{K}$.

Proof (i) Suppose a 3 -space $\Pi_{3}$ of the big 5 -space $\Pi_{5}$ intersects $\mathcal{K}$ in $q+2+x$ points, with $x \geq 0$. Then considering all 4-spaces in $\Pi_{5}$ through $\Pi_{3}$, we get at most $(2 q+1-(q+2+x))(q+1)+q+2+x=q^{2}+q+1-x q$ points in $\Pi_{5} \cap \mathcal{K}$ by Lemma 2.6, a contradiction if $x>0$. The second part follows directly if $x=0$.

(ii) By (i) there is a plane $\pi$ in $\Pi_{4}$ which intersects $\mathcal{K}$ in an oval. We claim we can find a second plane in $\Pi_{4}$ which intersects $\mathcal{K}$ in an oval. Take 3 points contained in $\Pi_{4} \cap \mathcal{K}$ not lying in $\pi$. These points span a plane $\pi^{\prime}$. The space $<\pi, \pi^{\prime}>$ is a 4 -space, otherwise we get a 3 -space intersecting $\mathcal{K}$ in more than $q+2$ points, contradicting (ii).

If $\pi^{\prime}$ contains exactly 3 points then consider all 3-spaces through $\pi^{\prime}$ in $\Pi_{4}$. If none of them contains at least 5 points we get at most $q+1+3=q+4$ points, a contradiction. So there is a 3 -space $\Pi_{3}$ through $\pi^{\prime}$ in $\Pi_{4}$ containing more than 4 points, hence by Conditions (P) and (S) we find a plane $\pi^{\prime \prime}$ containing $q+1$ points of $\mathcal{K}$ inside $\Pi_{3}$. Clearly $\pi$ and $\pi^{\prime \prime}$ are different since $\pi$ and $\pi^{\prime}$ span a 4 -space.

If the two different planes $\pi$ and $\pi^{\prime \prime}$ which intersect $\mathcal{K}$ in an oval intersect in a point, then we are done by Lemma 2.6. Suppose that $\pi$ and $\pi^{\prime \prime}$ intersect in a line. Then the 3 -space $\Pi_{3}^{\prime}=\left\langle\pi, \pi^{\prime \prime}\right\rangle$ intersects $\mathcal{K}$ in more than $q+2$ points, contradicting (ii).

Lemma 2.8 Every 4-space contained in a big 5-space $\Pi_{5}$ intersects $\mathcal{K}$ in $1, q+1$ or $2 q+1$ points and each such 5 -space contains at least one 4 -space intersecting $\mathcal{K}$ in exactly $2 q+1$ points. Hence, each big 5-space intersects $\mathcal{K}$ in a $\mathcal{V}_{2}^{4}$.

Proof Denote the number of points belonging to $\mathcal{K}$ contained in a 4-space $\Pi_{i} \subset \Pi_{5}$ by $x_{i}$; here $\Pi_{5}$ is a big 5 -space. In the following sum and all the others below, $i$ runs over all 4 -spaces $\Pi_{i}$ contained in $\Pi_{5}$. We have

$$
\sum_{i}\left(x_{i}-1\right)\left(x_{i}-(q+1)\right)\left(x_{i}-(2 q+1)\right)=0
$$


Indeed, by a standard counting technique counting in two different ways respectively the number of pairs $(p, \Pi)$ in $\Pi_{5}$, where $p \in \mathcal{K}$ and $\Pi$ is a 4 -space in $\Pi_{5}$, the number of triples $\left(p_{1}, p_{2}, \Pi\right), p_{1} \neq p_{2} \in \Pi \cap \mathcal{K}$ and $\Pi$ a 4 -space in $\Pi_{5}$, and the quadruples $\left(p_{1}, p_{2}, p_{3}, \Pi\right), p_{i} \in \Pi \cap \mathcal{K}$, where the points $p_{i}$ are all distinct and $\Pi$ is a 4 -space in $\Pi_{5}$ yields

$$
\begin{gathered}
\sum_{i} x_{i}=\frac{\left(q^{2}+q+1\right)\left(q^{5}-1\right)}{q-1} \\
\sum_{i} x_{i}\left(x_{i}-1\right)=\frac{\left(q^{2}+q+1\right)\left(q^{2}+q\right)\left(q^{4}-1\right)}{q-1} \\
\sum_{i} x_{i}\left(x_{i}-1\right)\left(x_{i}-2\right)=\frac{\left(q^{2}+q+1\right)\left(q^{2}+q\right)\left(q^{2}+q-1\right)\left(q^{3}-1\right)}{q-1} .
\end{gathered}
$$

If a 4 -space $\Pi_{4}$ inside a big 5 -space contains more than $q+1$ points of $\mathcal{K}$, then it is a big one. Indeed, by Lemma 2.6 there is a plane in $\Pi_{4}$ intersecting $\mathcal{K}$ in an oval. Hence we can find a 3 -space in $\Pi_{4}$ which intersects $\mathcal{K}$ in $q+2$ points. The claim now follows from (i) of Lemma 2.7.

Suppose $\left|\Pi_{4} \cap \mathcal{K}\right|=x, \Pi_{4} \subset \Pi_{5}$, with $4 \leq x<q+1$. Let $\Pi_{3}$ be a 3 -space containing 4 points $p_{1}, p_{2}, p_{3}, p_{4}$ in $\Pi_{4} \cap \mathcal{K}$. Hence, by Condition $(\mathrm{S}),\left|\Pi_{3} \cap \mathcal{K}\right|=4$. Consider all 4 -spaces through $\Pi_{3}$ inside $\Pi_{5}$. If there are less than 4 big ones among them, we get less than

$$
3(2 q-3)+(q-2)(q-3)+4=q^{2}+q+1
$$

points in $\Pi_{5} \cap \mathcal{K}$, a contradiction.

By Part (ii) of Lemma 2.7, in each of the at least 4 big 4 -spaces inside $\Pi_{5}$ containing $\Pi_{3}$ the points $p_{1}, p_{2}, p_{3}, p_{4}$ are contained in 2 ovals. Hence either there is an oval containing 3 of them, which yields a contradiction, or there is a pair $p_{i}, p_{j}$ contained in two different ovals. But the latter yields a contradiction by Part (i) of Lemma 2.7.

Suppose now that $\left|\Pi_{4} \cap \mathcal{K}\right|=3$. Consider a 3 -space $\Pi_{3}$ in $\Pi_{4}$ containing the 3 points $p_{1}, p_{2}, p_{3}$ of $\Pi_{4} \cap \mathcal{K}$ and all 4-spaces inside $\Pi_{5}$ containing $\Pi_{3}$. By the previous these intersect $\mathcal{K}$ in $3, q+1$ or $2 q+1$ points. Denote the number of them intersecting $\mathcal{K}$ in $q+1$ and $2 q+1$ points by $\alpha$ and $\beta$ respectively. This yields the following equation,

$$
\alpha(q-2)+\beta(2 q-2)+3=q^{2}+q+1 .
$$

We deduce that $\alpha$ is a multiple of $q-1$. If $\alpha=q-1$, then we get at most $(q-1)(q-$ $2)+2 q+1$ points in $\Pi_{5} \cap \mathcal{K}$, a contradiction.

Hence, we find that $\alpha=0$ and $\beta=\frac{q+2}{2}$. This already yields a contradiction if $q$ is odd. As $q>2$, the points $p_{1}, p_{2}, p_{3}$ are contained in 2 ovals in each of the at least 3 different big 4-spaces of $\Pi_{5}$ containing $\Pi_{3}$ by (ii) of Lemma 2.7. This yields a contradiction.

Finally, suppose that $\left|\Pi_{4} \cap \mathcal{K}\right|=2$. Consider a 3 -space $\Pi_{3}$ in $\Pi_{4}$ containing the 2 points $p_{1}, p_{2}$ of $\Pi_{4} \cap \mathcal{K}$ and all 4 -spaces inside $\Pi_{5}$ containing $\Pi_{3}$. By the previous these 
all intersect $\mathcal{K}$ in $2, q+1$ or $2 q+1$ points. Denote the number of them intersecting $\mathcal{K}$ in $q+1$ and $2 q+1$ points by $\alpha$ and $\beta$ respectively. This yields the following equation,

$$
\alpha(q-1)+\beta(2 q-1)+2=q^{2}+q+1 .
$$

This yields that $\beta-1$ is a multiple of $q-1$. If $\beta=1$, we get at most $(q-1)(q-1)+$ $2 q+1=q^{2}+2$ points of $\mathcal{K}$ in $\Pi_{5}$, a contradiction. If $\beta=q$, we get exactly $2 q^{2}-q+2$ points, also a contradiction.

In the previous paragraphs we proved that if a 4 -space contains at least 2 points of $\mathcal{K}$, then it contains at least $q+1$ points of $\mathcal{K}$. By (i) of Lemma 2.7 and (ii) of Lemma 2.6 the only possibilities in this case are $q+1$ and $2 q+1$. By Equation (1), this implies that there are no 4 -spaces which have an empty intersection with $\mathcal{K}$.

Hence, every 4 -space contained in $\Pi_{5}$ intersects $\mathcal{K}$ in $1, q+1$ or $2 q+1$ points.

We prove there is a 4 -space contained in $\Pi_{5}$ which intersects $\mathcal{K}$ in $2 q+1$ points. If this is not the case, then consider a 3 -space in $\Pi_{5}$ containing $x>1$ points of $\mathcal{K}$. We get the following equality:

$$
(q+1)(q+1-x)+x=q^{2}+q+1,
$$

hence $x=1$, a contradiction.

Hence by Theorem $1.3, \Pi_{5}$ intersects $\mathcal{K}$ in a Veronese variety $\mathcal{V}_{2}^{4}$.

Theorem 2.9 The set $\mathcal{K}$ is a Veronese variety $\mathcal{V}_{n}^{2^{n}}$.

Proof We check the conditions of Theorem 1.5. The set $\mathcal{P}$ consists of all planes intersecting $\mathcal{K}$ in an oval.

Any two points of $\mathcal{K}$ are contained in at least one oval of $\mathcal{K}$ by Condition $(\mathrm{P})$ and Lemma 2.4. If two points $p_{1}, p_{2}$ are contained in two ovals, namely $\mathcal{O}_{1}$ in the plane $\pi_{1}$ and $\mathcal{O}_{2}$ in the plane $\pi_{2}$, then these ovals span a 3 -space $\Pi_{3}$ containing too much points of $\mathcal{K}$, a contradiction. Indeed, consider a point $r$ on the intersection line $l$ of $\pi_{1}$ and $\pi_{2}$ which is not the nucleus of $\mathcal{O}_{1}$ neither of $\mathcal{O}_{2}$ and two bisecants $l_{1}$ and $l_{2}$ through $r$ to $\mathcal{O}_{1}$ and $\mathcal{O}_{2}$ respectively. Then the plane spanned by $l_{1}$ and $l_{2}$ contains at least 4 points of $\mathcal{K}$ and hence by Condition $(\mathrm{P}) q+1$ points of $\mathcal{K}$. In this way we get at least $2 q+q-3=3 q-3 \geq 2 q+2$ (since $q \geq 5$ ) points in $\Pi_{3} \cap \mathcal{K}$, a contradiction by Lemma 2.5 .

To prove Property (ii), consider two planes $\pi_{1}$ and $\pi_{2}$ which intersect $\mathcal{K}$ in an oval. If $\pi_{1} \cap \pi_{2}$ is a point then the property follows directly from Lemma 2.6. If $\pi_{1} \cap \pi_{2}$ is a line then we get a 3 -space $\Pi_{3}$ containing $2 q+1$ points. But then there are 4 -spaces through $\Pi_{3}$ containing more than $2 q+1$ points of $\mathcal{K}$, a contradiction.

For Property (iii), take a point $p$ not contained in a plane $\pi$ which intersects $\mathcal{K}$ in an oval $\mathcal{O}_{1}$. Consider two points $r$ and $s$ on $\mathcal{O}_{1}$ and the ovals $\mathcal{O}_{2}$ and $\mathcal{O}_{3}$ which are uniquely determined by $p$ and $r$ and $p$ and $s$ respectively. The point set of the ovals $\mathcal{O}_{1}, \mathcal{O}_{2}$ and $\mathcal{O}_{3}$ are contained in a 5 -space $\Pi_{5}$ intersecting $\mathcal{K}$ in more than $2 q+2$ points. Hence, by Lemma $2.8 \Pi_{5} \cap \mathcal{K}$ is a Veronesean $\mathcal{V}_{2}^{4}$. Take an arbitrary point $t$ on $\mathcal{O}_{1}$ and 
consider the oval determined by $p$ and $t$. Since $\Pi_{5} \cap \mathcal{K}$ is a $\mathcal{V}_{2}^{4}$, this oval is contained in $\Pi_{5}$. For each of these ovals there is a tangent at $p$ to these ovals. By Lemma 25.4.2 of [4] the union of these tangents forms a plane.

\section{Second characterization}

In this section, we show that for $n>2$, we can replace the set of conditions of Section 2 by the following set of conditions. Furthermore, we provide a counterexample for the case $n=2$.

Consider a set $\mathcal{K}$ of $\frac{q^{n+1}-1}{q-1}$ points spanning $\mathbf{P G}\left(\frac{n(n+3)}{2}, q\right)$, with $n>2$, such that

(P') If $\pi$ is a plane then the intersection $\pi \cap \mathcal{K}$ contains at most $q+1$ points of $\mathcal{K}$.

(S') If a 3 -space $\Pi_{3}$ intersects $\mathcal{K}$ in more than 4 points, then $\left|\Pi_{3} \cap \mathcal{K}\right| \geq q+1$ and $\Pi_{3} \cap \mathcal{K}$ is not a $(q+1)$-arc.

(V') If a 5 -space $\Pi_{5}$ intersects $\mathcal{K}$ in more than $2 q+2$ points then it intersects $\mathcal{K}$ in exactly $q^{2}+q+1$ points. Furthermore, any two points $p_{1}, p_{2}$ of $\mathcal{K}$ are contained in a 5 -space containing $q^{2}+q+1$ points of $\mathcal{K}$.

Lemma 3.1 Every 4 -space contains at most $2 q+2$ points of $\mathcal{K}$. Hence, a 3-space contained in a big 5-space contains at most $q+3$ points.

Proof Exactly the same as the proof of Lemma 2.5 using Condition (V'), since we only used there that part of Condition (V).

Lemma 3.2 For $n>2, q>5$, if a plane $\pi$ contains at least 4 points of $\mathcal{K}$ then it contains exactly $q+1$ points of $\mathcal{K}$.

Proof First suppose that $4<|\pi \cap \mathcal{K}|<q+1$. Then all 3-spaces through $\pi$ contain at least $q+1$ points. This yields at least $\frac{q^{\frac{n(n+3)}{2}-2}-1}{q-1}$ points for the set $\mathcal{K}$, a contradiction since $n>2$.

Next, suppose that $|\pi \cap \mathcal{K}|=4$. Consider points $a, b$ and $c$ of $\mathcal{K}$ such that $\langle\pi, a\rangle,\langle\pi, b\rangle$ and $\langle\pi, c\rangle$ are three different 3 -spaces. By Condition (S') each of these three 3-spaces intersects $\mathcal{K}$ in at least $q+1$ points. Then the space $\langle\pi, a, b, c\rangle$ contains at least $3(q-3)+4$ points of $\mathcal{K}$. Hence, if $q>7$, by Lemma 3.1 and Condition (V') it is a big 5 -space $\Pi_{5}$.

If $q=7$, we can always find a 3 -space containing at least 9 points of $\mathcal{K}$. Indeed otherwise inside a big 5 -space containing $\pi$, which always exists by the previous paragraph, we get the following equality: $4+4 \alpha=57$, a contradiction.

Consider a 3 -space $\Pi_{3}$ in $\Pi_{5}$ which contains $\pi$ and at least $q+1$ points of $\mathcal{K}$ or at least 9 in the case $q=7$. We show that $\left|\Pi_{3} \cap \mathcal{K}\right| \leq q+5$. Indeed, consider a second 
3 -space $\Pi_{3}^{\prime}$ in $\Pi_{5}$ which contains $\pi$ and at least $q+1$ points of $\mathcal{K}$. The 4 -space $\left\langle\Pi_{3}, \Pi_{3}^{\prime}\right\rangle$ intersects $\mathcal{K}$ in at least $\left|\Pi_{3} \cap \mathcal{K}\right|+\left|\Pi_{3}^{\prime} \cap \mathcal{K}\right|-4$ points. Since any 4 -space contains at most $2 q+2$ points of $\mathcal{K}$ by Lemma 3.1 the claim follows.

From the previous paragraph it follows that we get the following inequality for the number $x$ of 3 -spaces $\Pi_{3}^{i}$ through $\pi$ inside $\Pi_{5}$ containing at least $q+1$ points of $\mathcal{K}$.

$$
x(q+1)+4 \geq q^{2}+q+1 .
$$

Hence we get $x \geq q-\frac{3}{q+1}$, this implies $x \geq q$ if $q>2$.

Now consider a 3 -space $\Pi_{3}^{\prime \prime}$ containing $\pi$ and at least $q+1$ points of $\mathcal{K}$ which is not contained in $\Pi_{5}$ and consider also the 6 -space $\Pi_{6}=\left\langle\Pi_{5}, \Pi_{3}^{\prime \prime}\right\rangle$. Take one fixed 3 -space $\Pi_{3}^{1}$ and consider the 5 -spaces $\left\langle\Pi_{3}^{1}, \Pi_{3}^{\prime \prime}, \Pi_{3}^{i}\right\rangle$ with $i \neq 1$. Each of these 5 -spaces intersects $\mathcal{K}$ in more than $2 q+2$ points if $q>7$ and hence is a big 5 -space. It follows that $\Pi_{6} \cap \mathcal{K}$ contains at least $(q-1)\left(q^{2}-q-1\right)+2 q+2=q^{3}-2 q^{2}+2 q+3$ points.

Repeating this reasoning yields inductively the following recursion formula for the number of points $\phi_{k+1}$ in $\Pi_{k+1} \cap \mathcal{K}$ where $\tilde{\Pi}_{3}$ is a 3 -space containing $\pi$ and at least $q+1$ points of $\mathcal{K}$ which is not contained in $\Pi_{k}$ and where $\Pi_{k+1}=\left\langle\Pi_{k}, \tilde{\Pi}_{3}\right\rangle$, where $\phi_{5}=q^{2}+q+1$.

$$
\phi_{k+1}=\left(\frac{\phi_{k}-4}{q+1}-1\right)\left(q^{2}-q-1\right)+2 q+2 .
$$

We will adapt the recursion formula to a recursion formula for numbers $\psi_{k}$ such that $\psi_{k} \leq \phi_{k}$ for all $k \geq 5$.

First we rewrite the recursion formula for $\phi_{k}$ as follows.

$$
\phi_{k+1}=\left(\phi_{k}-q-5\right) \frac{q^{2}-q-1}{q+1}+2 q+2
$$

Since $\frac{q^{2}-q-1}{q+1}>q-2$ we get after a little calculation

$$
\phi_{k+1}>(q-2) \phi_{k}-q^{2}-q+8 \text {. }
$$

Since $\phi_{5}=q^{2}+q+1$ we can even write for all integers $k \geq 5$

$$
\phi_{k+1}>(q-3) \phi_{k} .
$$

Now we set $\psi_{5}=\phi_{5}$ and $\psi_{k+1}=(q-3) \psi_{k}$. Hence we get $\psi_{N}=(q-3)^{N-5}\left(q^{2}+q+1\right)$ for all $N \geq 5$. This yields the following inequality

$$
(q-3)^{\frac{n(n+3)}{2}-5}\left(q^{2}+q+1\right) \leq \frac{q^{n+1}-1}{q-1} .
$$

This is an equality if $n=2$ and the left hand side increases faster than the right hand side if $n$ increases, hence this yields a contradiction for $n>2$.

The case remaining is $q=5$. 
Lemma 3.3 For $q=5$ a plane $\pi$ intersecting $\mathcal{K}$ in exactly 4 points is never contained in a big 5-space $\Pi_{5}$.

Proof First of all, a 3 -space in $\Pi_{5}$ contains at most 8 points of $\mathcal{K}$ by Lemma 3.1.

Project everything from $\pi$ onto a plane $\pi^{\prime}$ which is skew to $\pi$ in $\Pi_{5}$. With the 3 -spaces through $\pi$ which contain 6,7 or 8 points of $\mathcal{K}$, we associate a point in $\pi^{\prime}$ with weight 2,3 or 4 respectively.

First suppose there is a 3 -space $\Pi_{3}$ in $\Pi_{5}$ which contains $\pi$ and which intersects $\mathcal{K}$ in 8 points. Then five 4 -spaces through $\Pi_{3}$ in $\Pi_{5}$ intersect $\mathcal{K}$ in 12 points and one 4 -space through $\Pi_{3}$ in $\Pi_{5}$ intersects $\mathcal{K}$ in 11 points. This implies that inside a big 5 -space through $\Pi_{3}$ there is exactly one 3 -space $\Pi_{3}^{\prime}$ which contains $\pi$ and which intersects $\mathcal{K}$ in 7 points.

But a 4 -space $\Pi_{4}$ through $\Pi_{3}$ intersecting $\mathcal{K}$ in 12 points has to be contained in at least one other big 5 -space $\tilde{\Pi}_{5}$. Inside $\tilde{\Pi}_{5}$ we also find a 3 -space $\tilde{\Pi}_{3}$ containing 7 points of $\mathcal{K}$. But now the big 5 -space spanned by $\Pi_{3}, \Pi_{3}^{\prime}$ and $\tilde{\Pi}_{3}$ contains $\Pi_{3}$ and two 3 -spaces which contain $\pi$ and which intersect $\mathcal{K}$ in 7 points, a contradiction by the previous paragraph.

Hence, from now on, we may assume that each 3 -space through $\pi$ inside a big 5 -space which contains more than 4 points of $\mathcal{K}$ contains 6 or 7 points of $\mathcal{K}$. Hence if we denote the number of 3 -spaces through $\pi$ inside $\Pi_{5}$ which intersect $\mathcal{K}$ in 6 points by $\alpha$ and those which intersect $\mathcal{K}$ in 7 points by $\beta$, we get the following equation,

$$
4+2 \alpha+3 \beta=31 \text {. }
$$

The rest of the proof is case-by-case analysis.

(A) $\beta \geq 7$ :

In this case we have a set $\mathcal{P}$ of 7 points with weight 3 in $\pi^{\prime}$. Since an oval in $\mathbf{P G}(2,5)$ contains at most 6 points, three points of $\mathcal{P}$ will be contained in the same line $L$. But this implies that the 4 -space spanned by $L$ and $\pi$ intersects $\mathcal{K}$ in more than 12 points, a contradiction by Lemma 3.1 .

(B) $\alpha=6, \beta=5$ :

Consider a point $p$ of weight 3 in $\pi^{\prime}$ and all lines through it. On four of these lines we have exactly one other point which has weight 3 otherwise we get a 4 -space with more than 12 points. But then the 6 points of weight 2 have to be distributed over the remaining 2 lines, which again yields a too big 4-space.

(C) $\alpha=9, \beta=3$ :

Consider a point $p$ of weight 3 in $\pi^{\prime}$ and all lines through it. On two of these lines we have exactly one other point which has weight 3 otherwise we get a 4 -space with more than 12 points. But then the 9 points of weight 2 have to be distributed over the remaining 4 lines, which again yields a too big 4 -space.

(D) $\alpha=12, \beta=1$ : 
Denote the 3 -space through $\pi$ inside $\Pi_{5}$ which intersects $\mathcal{K}$ in 7 points by $\Pi_{3}$. We have a set $\mathcal{P}$ of 13 points in $\pi^{\prime}$. We claim that there has to be a line $L$ containing 4 points of $\mathcal{P}$.

Indeed, consider an arbitrary point $p$ contained in $\mathcal{P}$ and all lines through it. If there is no line among them which intersects $\mathcal{P}$ in 4 points then they all intersect $K$ in 3 points. Since $p$ was arbitrary this implies that all lines in $\pi^{\prime}$ intersect $\mathcal{P}$ in 0 or 3 points. But now consider a point $r$ in $\pi^{\prime}$ not contained in $\mathcal{P}$ and all lines through it. Then we get a contradiction, since 3 does not divide 13 .

Hence, the fourspace spanned by $L$ and $\pi$ intersects $\mathcal{K}$ in 12 points. It has to be contained in another big 5-space otherwise we don't get enough points in $\mathcal{K}$. There again there has to be at least one 3 -space, say $\Pi_{3}^{\prime}$, through $\pi$ which intersects $\mathcal{K}$ in 7 points.

But now consider a space $\hat{\Pi}$ spanned by $\Pi_{3}, \Pi_{3}^{\prime}$ and another 3 -space which contains at least 6 of $\mathcal{K}$ through $\pi$.

Then $\hat{\Pi}$ is certainly contained in a big 5 -space, otherwise we don't have enough points in the set $\mathcal{K}$ but this is a contradiction since in any big 5 -space we already excluded all cases with $\beta>1$.

Lemma 3.4 Any line $l$ meets $\mathcal{K}$ in at most 2 points. Hence, a plane $\pi$ with $|\pi \cap \mathcal{K}|=$ $q+1$ intersects $\mathcal{K}$ in an oval.

Proof Similar to the proof of Lemma 2.4; use Lemma 3.1 and Lemma 3.2.

Theorem 3.5 If $q \geq 5$ and $n>2$, then the set $\mathcal{K}$ is the point set of the Veronese variety of all quadrics of $\mathbf{P G}(n, q)$.

Proof We check Conditions (P), (S) and (V) of Theorem 2.1. Conditions (S) and $(\mathrm{V})$ are trivially implied by Conditions $\left(\mathrm{S}^{\prime}\right)$ and $\left(\mathrm{V}^{\prime}\right)$ respectively. The first part of Condition (P) was proved in Lemma 3.2 for $q>5$.

Furthermore, for $q=5$ we proved Condition $(\mathrm{P})$ for all planes which are contained in a big 5-space. In fact, we did only use Condition $(\mathrm{P})$ for these plane in our first characterization.

The second part of Condition $(\mathrm{P})$, namely that every 2 points of $\mathcal{K}$ are contained in an oval of $\mathcal{K}$, is never used to obtain Lemma 2.8 if $n>2$. If $n=2$, we did use Condition (P) for the proof of Lemma 2.5. Since every two points are contained in a big 5-space $\Pi_{5}$ by Condition $(\mathrm{V})$, and since $\Pi_{5} \cap \mathcal{K}$ is a Veronesean surface $\mathcal{V}_{2}^{4}$ the second part of Condition (P) is proved. The proof is finished by Theorem 2.1.

The counterexample for the case $n=2$ is the following. Consider in $\mathbf{P G}(5, q)$ a point $p$ on an ovoid $\mathcal{O}$ in $\operatorname{PG}(3, q)$ and a tangent line $L$ to $\mathcal{O}$ at $p$. Furthermore, consider a second 3 -space $\Pi_{3}^{\prime}$ intersecting $\Pi_{3}$ exactly in $L$ and containing an oval $\mathcal{O}^{\prime}$ which intersects $L$ in $p$. Then the set $\mathcal{O} \cup \mathcal{O}^{\prime}$ fulfills Conditions ( $\left.\mathrm{P}^{\prime}\right),\left(\mathrm{S}^{\prime}\right)$ and (V') but it is not a Veronesean $\mathcal{V}_{2}^{4}$. 
Acknowledgement The research of the first author takes place within the project "Linear codes and cryptography" of the Fund for Scientific Research Flanders (FWOVlaanderen) (Project nr. G.0317.06), and is supported by the Interuniversitary Attraction Poles Programme-Belgian State-Belgian Science Policy: project P6/26-Bcrypt. Address of the authors:

Ghent University, Dept. of Pure Mathematics and Computer Algebra, Krijgslaan 281-S22, 9000 Ghent, Belgium

J. Schillewaert: jschille@cage.ugent.be

J.A. Thas: jat@cage.ugent.be, http://cage.ugent.be/ jat

H. Van Maldeghem: hvm@cage.ugent.be, http://cage.ugent.be/ hvm

\section{References}

[1] A. Del Fra, On d-dimensional dual hyperovals, Geom. Dedicata, 79, (2000), $157-178$.

[2] O. FerRI, Su di una caratterizzazione grafica della superficie di Veronese di un $S_{5, q}$, Atti Accad. Naz. Lincei Rend., 61 (1976), 603-610.

[3] J. W. P. Hirschfeld. Finite projective spaces of three dimensions, Second edition. Oxford University Press, Oxford, 1985, 316pp.

[4] J. W. P. Hirschfeld and J. A. Thas, General Galois Geometries, Oxford University Press, Oxford, 1991.

[5] B. Segre, Ovals in a finite projective plane, Canad. J. Math., 7 (1955), 414-416.

[6] J. A. Thas and H. Van Maldeghem, Characterizations of the finite quadric Veroneseans $\mathcal{V}_{n}^{2^{n}}$, Quart. J. Math., 55 (2004), 99-113.

[7] J. A. Thas and H. Van Maldeghem, Classification of finite Veronesean caps, European J. Combin., 25 (2004), 275-285.

[8] C. Zanella, Linear sections of the finite Veronese varieties and authentication systems defined using geometry, Des. Codes Cryptogr., 13 (1998), 199-212. 